\section{OBSTRUCTIVE SLEEP APNEA AND OFFICE- BASED SURGERY}

Obstructive sleep apnea (OSA) is a recognized risk factor for complications during anesthesia during both the intraoperative and the postoperative periods. Unfortunately, only a small subset of patients who have OSA are formally diagnosed with the condition. There are many reasons, maybe chief among them being that the gold standard for testing is overnight polysomnography, which is expensive and inconvenient. The apneahypopnea index (AHI; number of apneas $>10$ seconds and hypopneas per hour) is frequently used to assess the severity of OSA. An AHI value $\geq 30$ events/h reflects severe OSA. Moderate OSA is defined by the AHI as ranging between 15 and 29.99 events/h and mild OSA between 5 and 14.99 events/h. Although home-based monitoring is available, its reliability has not been clearly validated and the cost is high.

Among the risks of OSA during the perioperative period, upper-airway obstruction, hypertensive episodes, dysrhythmia, and more serious events such as myocardial infarction and stroke are some of the hazards that may be encountered. In the dental office setting, the natural airway is still the most common method of airway management for sedation and general anesthesia. Upper-airway obstruction leading to hypoxemia and hypercarbia may precipitate all of the above adverse events. Furthermore, patients with OSA tend to be exquisitely sensitive to opioids such that even small doses may provoke significant respiratory depression. ${ }^{1}$ This concern extends into the postoperative period, where opioids may be prescribed for postoperative pain.

Screening for OSA has become standard in most hospital and surgery center anesthesia departments for this reason. Therefore, some method of screening for OSA in the dental office would seem prudent. In the hospital/ambulatory surgery center (ASC) settings, if a natural airway is selected for sedation, most anesthesia providers are comfortable changing the anesthesia plan to an intubated or laryngeal mask airway. This is likely not the case with most deep-sedation providers in the dental office setting. Moderate-sedation providers, assuming they are using only benzodiazepines and/or opioids, can consider partial or full reversal should management of airway obstruction with attempted arousal and other airway maneuvers not be successful.

Recognition of those patients at risk of OSA would allow the dental sedation/general anesthesia provider to consider whether a patient with suspected, but not formally diagnosed, OSA should have a modified sedation/anesthesia plan or if he or she is even

Anesth Prog 63:53-54 2016

(C) 2016 by the American Dental Society of Anesthesiology appropriate for the office setting based on patient and procedural needs. There are a number of validated screening tools, each with fair to good positive and negative predictive values. The first widely used screening tool was the STOP questionnaire. ${ }^{2}$ This instrument assesses the presence of 4 symptoms: Snoring, daytime Tiredness, Observed apnea by a bed partner, and high blood Pressure. Later, the STOP-Bang questionnaire (see Appendix) combined 4 other criteria with the STOP questionnaire: Body mass index (BMI) $>35 \mathrm{~kg} / \mathrm{m}^{2}$, age $>50$ years, neck circumference $>40 \mathrm{~cm}(15.75 \mathrm{in})$, and male gender. ${ }^{2}$ The STOP-Bang (see below) has good specificity and sensitivity for detecting AHI $\geq 30$. Recently, a new instrument has been developed, the DES-OSA, that includes only Mallampati score, BMI, neck circumference, distance from chin to thyroid cartilage with the head in neutral position, and sex. ${ }^{3}$ Early studies reveal good sensitivity and specificity for $\mathrm{AHI} \geq 30$ as well. Interestingly, the American Dental Association is considering adding BMI to the required documentation for sedation patients, so many of these data may already be collected.

As helpful as these instruments are, all anesthesia providers have encountered deep-sedation/nonintubated general anesthesia patients who appear to have good airways, only to be confronted with an easily obstructed airway that is difficult to manage. I recently encountered just such a patient whose airway did not improve with insertion of a standard nasopharyngeal airway or with a custom-cut endotracheal tube used as a long nasopharyngeal airway. The case was converted to an intubated general anesthetic and the patient safely managed.

What can we do as sedation/anesthesia providers to maximize quality care in office-based sedation? A thorough medical history and a standard physical examination are critical elements of the preoperative evaluation that help ensure safety. A thorough airway examination, including application of screening tests such as STOP-Bang, can improve our ability to recognize those patients who are at increased risk for office-based sedation/general anesthesia. It should be appreciated that not all patients are suitable for sedation/general anesthesia in the dental office. Likewise, not every sedation/general anesthesia provider can manage every patient that needs office-based sedation. Just as a well-trained ENT surgeon may choose to refer a difficult head and neck cancer reconstruction patient to another ENT or oral and maxillofacial surgeon, moderate-sedation providers and oral and maxillofacial surgeons may choose to engage the services of a comprehensively trained anesthesia provider in the office setting or hospital/ASC for some patients. This should not SSDI 0003-3006(16) 
be viewed as an inadequacy or a failure. Providing the best and safest service we can for our patients should always be our primary practice goal.

Steven Ganzberg, DMD, MS

\section{REFERENCES}

1. Brown KA, Laferriere A, Moss IR. Recurrent hypoxemia in young children with obstructive sleep apnea is associated with reduced opioid requirement for analgesia. Anesthesiology. 2004;100:806-810.

2. Chung F, Yegneswaran B, Liao P, et al. STOP questionnaire: a tool to screen patients for obstructive sleep apnea. Anesthesiology. 2008;108:812-821.

3. Deflandre E, Degey S, Brichant JF, Poirrier R, Bonhomme $\mathrm{V}$. Development and validation of a morphologic obstructive sleep apnea prediction score: the DES-OSA score. Anesth Analg. 2016;122:363-372.

\section{APPENDIX}

\section{STOP-BANG Sleep Apnea Questionnaire}

Chung $F$ et al Anesthesiology 2008 and BJA 2012

\begin{tabular}{|l|c|c|}
\hline \multicolumn{1}{|c|}{ STOP } & & \\
\hline $\begin{array}{l}\text { Do you SNORE loudly (louder than talking or loud } \\
\text { enough to be heard through closed doors)? }\end{array}$ & Yes & No \\
\hline $\begin{array}{l}\text { Do you often feel TIRED, fatigued, or sleepy during } \\
\text { daytime? }\end{array}$ & Yes & No \\
\hline $\begin{array}{l}\text { Has anyone OBSERVED you stop breathing during } \\
\text { your sleep? }\end{array}$ & Yes & No \\
\hline $\begin{array}{l}\text { Do you have or are you being treated for high blood } \\
\text { PRESSURE? }\end{array}$ & Yes & \\
\hline
\end{tabular}

\begin{tabular}{|l|c|c|}
\hline \multicolumn{1}{|c|}{ BANG } & & \\
\hline BMI more than $35 \mathrm{~kg} / \mathrm{m} 2$ ? & Yes & No \\
\hline AGE over 50 years old? & Yes & No \\
\hline NECK circumference $>16$ inches $(40 \mathrm{~cm}) ?$ & Yes & No \\
\hline GENDER: Male? & Yes & No \\
\hline
\end{tabular}

\section{TOTAL SCORE}

High risk of OSA: Yes 5 - 8

Intermediate risk of OSA: Yes 3 - 4

Low risk of OSA: Yes 0 - 2 